Review article

\title{
The new criteria for classification of rheumatoid arthritis: what we need to know for clinical practice ${ }^{\text {ts }}$
}

\author{
Salvatore Corrao *, Luigi Calvo, Giuseppe Licata \\ Dipartimento Biomedico di Medicina Interna e Specialistica, University of Palermo, Italy
}

\section{A R T I C L E I N F O}

\section{Article history:}

Received 4 December 2010

Received in revised form 19 December 2010

Accepted 22 December 2010

Available online 26 January 2011

\section{Keywords:}

Rheumatoid arthritis

Classification criteria

Anti-citrullinated peptide autoantibodies

Bayesian reasoning

Likelihood ratio

Sensitivity and specificity

\begin{abstract}
A B S T R A C T
The new criteria for classification of Rheumatoid Arthritis have been recently released. They incorporate the anti-Citrullinated Protein antibody testing and the other classic criteria in a score system (the diagnosis of definite rheumatoid arthritis is made by a total score $\geq 6$ ). These criteria try to meet the pressing needs to gain sensitivity in early disease. Symptoms, elevated acute-phase response, serologic abnormality, joint involvement were all considered for scoring after confirming the presence of synovitis in at least 1 joint in the absence of an alternative diagnosis that better explains the synovitis. However, no sensitivity and specificity has been showed. Moreover, Area Under Curve of the Receiver Operating Characteristic curves (a measure of performance of the test) was not optimal in almost two of the three studied cohorts. On the contrary, the old criteria of the American College of Rheumatology had been tested to calculate sensitivity and specificity. Moreover, sensitivity and specificity of anti-citrullinated peptide auto-antibodies are available for clinical reasoning based on pre-test and post-test probabilities of the disease. The use of likelihood ratios applied to both the old criteria and anti-citrullinated autoantibodies could help clinicians to effectively manage early arthritis patients implementing Bayesian reasoning. Here, we tried to explain the methodology applied to the body of knowledge currently available about rheumatoid arthritis for diagnostic decisionmaking based on the Bayesian approach.
\end{abstract}

(C) 2011 European Federation of Internal Medicine. Published by Elsevier B.V. All rights reserved.
The 2010 Rheumatoid Arthritis classification criteria were recently published [1]. They try to meet the pressing needs to gain sensitivity in early disease. Symptoms, elevated acute-phase response, serologic abnormality, joint involvement were all considered for scoring after confirming the presence of synovitis in at least 1 joint in the absence of an alternative diagnosis that better explains the synovitis (Table 1). Anti-Citrullinated Protein antibody testing was incorporated for the first time into the new diagnostic criteria. However, various issues should be addressed. First of all, the paper does not report any sensitivity and specificity of the new criteria whereas the old criteria had reported these values. The whole formulation process was based on a form of structured consent by expert opinion. On the contrary, since three large cohorts were available, a statistically driven process could be used to weight each criterion by likelihood ratios. Positive and negative likelihood ratios (and their 95\% Confidence Intervals) could

\footnotetext{
is This manuscript had no financial support or other benefits from commercial sources. Moreover, all the authors have not any financial interests that could create a potential conflict of interest or the appearance of a conflict of interest with regard to this work.

* Corresponding author. Laboratory of Clinical Epidemiology, Biostatistics and Knowledge Management, Dipartimento Biomedico di Medicina Interna e Specialistica, University of Palermo [Biomedical Department of Internal Medicine and Subspecialties], Piazza delle Cliniche 2, 90147 Palermo, Italy. Tel.: +30 091 6552065; fax: +39 091 6552165.

E-mail address: s.corrao@tiscali.it (S. Corrao).
}

be computed for groups with similar criteria. Thus, the property of combining independent likelihood ratios could have been used for implementation in clinical practice. Unfortunately, for these reasons, the opportunity to put into practice an intriguing evidence based approach has been missed out. Last but not least, the great effort that was made seems to have brought forth a mouse. If, on one hand, either sensitivity and specificity or likelihood ratios were not reported, on the other hand, the overall accuracy seems poor. In particular, the Area Under Curve for Receiver Operating Characteristic curves (which plot sensitivity, or true positive rate, against 1-specificity, or false positive rate, to individuate the best discriminating cut-off value of a quantitative variable, as a score is) resulted good in the Norwegian cohort (0.88) but insufficient in both the French and Rotterdam ones $(<0.70)$. Even though they were always statistically significant, notable variability among the Receiver Operating Characteristic curves of the three different cohorts raises many doubts about clinical significance. However, clinicians need clinically significant tools for clinical practice where statistical significance is only a necessary but not a sufficient criterion. On the other hand, the old criteria were developed in the 1987 revision using the method of classification trees. This analysis pointed out a specificity of $89 \%$ and a sensitivity of about $84 \%$ for the rule requiring 4 out of 7 American College of Rheumatology criteria. Moreover, in recent years, the anti-Citrullinated Peptide autoantibodies have emerged as valuable serologic marker of Rheumatoid Arthritis while Rheumatoid Factor is the only biomarker 
Table 1

The 2010 Classification Criteria for Rheuamtoid Arthritis (score is intended to be computed on patients who have at least 1 joint with definite clinical synovitis (joint swelling) without any other disease explanation).

Score

$\begin{array}{ll}\text { A. Joint involvement } & \\ \text { a. large joint. } & 0 \\ \text { b. } 2-10 \text { large joints } & 1 \\ \text { c. } 1-3 \text { small joints (with or without involvement of large joints) } & 2 \\ \text { d. } 4-10 \text { small joints (with or without involvement of large joints) } & 3 \\ \text { e. } \geq 10 \text { joints (at least } 1 \text { small joint) } & 5 \\ \text { B. Serology (at least } 1 \text { test result is needed for classification) } & \\ \text { a. Negative RF and negative ACPA } & 0 \\ \text { b. Low-positive RF or low-positive ACPA } & 2 \\ \text { c. High-positive RF or high-positive ACPA } & 3 \\ \text { C. Acute-phase reactants (at least } 1 \text { test result is needed for classification) } \\ \begin{array}{l}\text { a. Normal CRP and normal ESR } \\ \text { b. Abnormal CRP or abnormal ESR }\end{array} \\ \begin{array}{l}\text { D. Duration of symptoms } \\ \text { a. }<6 \text { weeks }\end{array} \\ \text { b. } \geq 6 \text { weeks } & 1 \\ \end{array}$

"Large joints" refers to shoulders, elbows, hips, knees, and ankles.

"Small joints" refers to the metacarpophalangeal joints, proximal interphalangeal joints, second through fifth metatarsophalangeal joints, thumb interphalangeal joints, and wrists.

For more details, please refer to the main publication (1).

The diagnosis of definite rheumatoid arthritis is made by a total score $\geq 6$.

RF: Rheumatoid Factor; ACPA: Anti-Citrullinated Peptide Autoantibodies; CRP:C Reactive Protein; ESR: Erythrocyte Sedimentation Rate.

considered by the above-mentioned old American College of Rheumatology criteria. The importance of anti-Citrullinated Peptide autoantibodies consists in the best specificity in comparison with Rheumatoid Factor in patients affected by early Rheumatoid Arthritis $[2,3]$. Recently, a systematic review has been published on accuracy of anti-Citrullinated Peptide autoantibodies for diagnosing early RA [4]. This analysis of 151 studies showed that sensitivity and specificity were $57 \%$ (95\% Confidence Intervals, 51\% to 63\%) and 96\% (93\% to 97\%), respectively (analysis refers only to 15 relevant cohort studies). Thus, sensitivity and specificity of the old American College Rheumatoid criteria are available to us as much the same data for anti-Citrullinated Peptide antibodies. How can this information be utilized for clinical practice? Sensitivity and specificity are stable characteristic of a test. The Bayes' theorem demonstrates that the Likelihood Ratio of both a positive and negative test allows to compute posterior probability using different "a priori" probabilities [5]. In a few words Likelihood Ratios are sufficiently stable characteristic of a test with more informative power than sensitivity and specificity (usually cryptic information for the clinician). Indeed, sensitivity and specificity are useful parameters when they reach values near $100 \%$ (a negative result of a test with sensitivity of $100 \%$ permits to exclude a disease while a test with specificity of $100 \%$ is highly indicative of disease when it results positive). On the contrary, Likelihood Ratio of a positive test and of a negative one are always informative and easily usable. Generally, a positive Likelihood Ratio $>15$ suggests a good probability to diagnose a disease while a negative Likelihood Ratio $<0.15$ suggests a good probability to exclude a disease [6]. However, Likelihood Ratios have another very interesting characteristic: their values can be multiplied between or among Likelihood Ratios for different tests. For example, if we have two independent tests for a disease (antiCitrullinated Peptide antibodies and the old American College of Rheumatology criteria are the case) we can multiply the positive Likelihood Ratios by themselves. The old American College of Rheumatology criteria have a positive Likelihood Ratio of 8.54 and a negative Likelihood Ratio of 0.07 (computed from the original sensitivity and specificity values of the American College of Rheumatology criteria). Anti-Citrullinated Peptide antibodies have a positive Likelihood Ratio of 14 and a negative Likelihood Ratio of 0.45 (data computed from the above-mentioned systematic review). It is obvious that none of the two diagnostic tests is easily usable in clinical practice for diagnosing or excluding Rheumatoid arthritis. However if we combine their values (positive Likelihood Ratios $=8.54 \times 14=120$; negative Likelihood Ratios $=0.07 \times 0.45=0.031$ ) Likelihood Ratios (positive and negative) became highly informative for an unexperienced physician as well. Even if we consider their 95\% Confidence Intervals remain highly informative and usable in different clinical practice settings (e.g. general practitioner setting or a rheumatologic outpatient clinic). Moreover, the use of combined information simplifies diagnostic utilization of anti-Citrullinated Peptide autoantibody cut-off values. Indeed, Pietrapertosa et al [7], in another recent paper, have correctly pointed out the importance of different cut-off values to modify Likelihood Ratios according to Sackett lessons [6]. Indeed, different cut-off values can generate the so-called SPin (Specificity In: high values for the highest specificity, consequently to diagnose disease) and SNout (Sensitivity Out: low values for the highest sensitivity, consequently to exclude disease). Indeed, high anti-Citrullinated Peptide autoantibody cut-off value $(>15.0$ or $>30.0 \mathrm{U} / \mathrm{mL}$, that correspond to positive Likelihood Ratios of 42 and infinity, respectively) could became very useful to diagnose early RA much precociously, that is, when both American College of Rheumatology criteria cannot be applicable (onset of symptoms $<6$ week) or only one criterion is lacking to get a positive result.

However, as shown above, we can simply use two diagnostic test (anti-Citrullinated Peptide autoantibodies and the old American College of Rheumatology criteria) to easily reach diagnostic values of positive or negative Likelihood Ratios.

Archibald Cochrane claimed needs for synthesis caused by information overload [8]. The Cochrane network systematic reviews have become the standard to summarize information toward knowledge growth [9]. Now, we also need to organize information to generate usable knowledge using both the available methodology from clinical epidemiology and clinical research data [10]. We believe that what we have explained in this manuscript goes toward this direction. Scientific community and prominent journals oriented to readership of clinical practitioners must continue to provide knowledge support for ease clinical practice implementation. We strongly claim that scientific knowledge had to be based on facts and not on faith.

\section{Learning points}

- The new criteria for classification of Rheumatoid Arthritis incorporate the anti-Citrullinated Protein antibody testing and the other classic criteria in a score system. The diagnosis of definite rheumatoid arthritis is made by a total score $\geq 6$. These criteria might be more useful for classification of patients with early arthritis disease. However, no sensitivity and specificity has been showed. Moreover, Area Under Curve of the Receiver Operating Characteristic curves (a measure of performance of the test) was not optimal in almost two of the three studied cohorts.

- On the contrary, the old criteria of the American College of Rheumatology had been tested to calculate sensitivity and specificity. Moreover, sensitivity and specificity of anti-citrullinated peptide auto-antibodies are available for clinical reasoning based on pre-test and post-test probabilities of the disease. The use of likelihood ratios (see the text) applied to both the old criteria and anti-citrullinated autoantibodies could help clinicians to effectively manage early arthritis patients implementing Bayesian reasoning.

- Less than four of the old classification criteria and negative anticitrullinated peptide autoantibodies allow to exclude rheumatoid arthritis.

- Even If a patient had not sufficient probability of disease (below four of the old criteria) but the anti-citrullinated peptide autoantibodies $>15 \mathrm{U} / \mathrm{mL}$, the diagnosis of rheumatoid arthritis could be set. 


\section{References}

[1] Aletaha D, Neogi T, Silman AJ, Funovits J, Felson DT, Bingham 3rd CO, et al. American College of Rheumatology; European League Against Rheumatism. 2010 Rheumatoid arthritis classification criteria: an American College of Rheumatology/ European League Against Rheumatism collaborative initiative. Arthritis Rheum 2010;62(9):2569-81.

[2] Schellekens GA, Visser H, de Jong BA, van den Hoogen FH, Hazes JM, Breedveld FC, et al. The diagnostic properties of rheumatoid arthritis antibodies recognizing a cyclic citrullinated peptide. Arthritis Rheum 2000;43:155-63.

[3] Nishimura K, Sugiyama D, Kogata Y, Tsuji G, Nakazawa T, Kawano S, et al. Metaanalysis: diagnostic accuracy of anticyclic citrullinated peptide antibody and rheumatoid factor for rheumatoid arthritis. Ann Intern Med 2007;146:797-808

[4] Whiting PF, Smidt N, Sterne JA, Harbord R, Burton A, Burke M, et al. Systematic review: accuracy of anti-citrullinated Peptide antibodies for diagnosing rheumatoid arthritis. Ann Intern Med 2010;152(7):456-64.
[5] Corrao S, Pistone G. Practical Manual for diagnosing in rheumatology: Bayesian reasoning according to ACR diagnostic criteria. Roma: Pensiero Scientifico Editore; 2009

[6] Sackett DL, Haynes RB, Guyatt GH, Tugwell P. Clinical Epidemiology: a basic science for clinical medicine. Boston: Little Brown; 1991.

[7] Pietrapertosa D, Tolusso B, Gremese E, Papalia MC, Bosello SL, Peluso G, et al. Diagnostic performance of anti-citrullinated peptide antibodies for the diagnosis of rheumatoid arthritis: the relevance of likelihood ratios. Clin Chem Lab Med 2010;48(6):829-34.

[8] Cochrane AL. Effectiveness and Efficiency: Random Reflections of Health Services. London: Nuffield Provincial Hospitals Trust; 1971.

[9] http://www.cochrane.org/cochrane-reviews last access on december 132010.

[10] Corrao S, Arcoraci V, Arnone S, Calvo L, Scaglione R, Di Bernardo C, et al. EvidenceBased Knowledge Management: an approach to effectively promote good healthcare decision-making in the Information Era. Intern Emerg Med 2009;4(2): 99-106. 